\title{
Micro-Machined Heat Pipes in Silicon MCM Substrates
}

\author{
D. A. Benson, R. T. Mitchell, M. R. Tuck, D. R. Adkins, and D. W. Palmer \\ Sandia National Laboratories, Albuquerque, NM
}

\begin{abstract}
Multichip modules (MCMs) containing power components need a substrate with excellent heat spreading capability both to avoid hot spots and to move dissipated heat toward the system heat sinks. Polycrystalline diamond is an excellent MCM heat spreading substrate but remains several orders of magnitude too expensive and somewhat more difficult to process than conventional mother-board materials. Today's power MCMs concentrate on moderately priced silicon wafers and aluminum nitride ceramic with their improved thermal conductivity and good thermal expansion match to power semiconductor components, in comparison to traditional alumina and printed wiring board materials. However, even silicon and AlN substrates are challenged by designers' thermal needs. We report on the fabrication of micro-heat pipes embedded in silicon MCM substrates (5x5 cm) by the use of micromachined capillary wick structures and hermetic micro-cavities. This passive microstructure results in more than a 5 times improvement in heat spreading capability of the silicon MCM substrate over a large range of power densities and operating temperatures as compared with silicon alone. Thus diamond-like cooling is possible at silicon prices. .
\end{abstract}

\section{Motivation}

Substrates with higher thermal conductivity are continually under development to meet the demand of increasing power density on modules. For example, considerable effort in polycrystalline diamond [1] and AlN ceramic are underway today. In addition, several companies market metal cold plates which transfer heat from a substrate through heat pipes to a remote condenser unit, all within a desktop computer.

After much development, the cost of a one-inch-square diamond substrate is still in the $\$ 300-\$ 1000$ range. In addition, cost scales much faster than substrate area. Heat pipes intended for electronics cooling are scaled down versions of larger designs of the past but still are relatively

This work was performed by Sandia National Laboratories with funding from the United States Department of Energy under Contract DE-AC04-94AL85000. large compared to the working substrate to be cooled. To overcome the cost and size difficulties, Sandia Labs pursued a concept of embedding micromachined heat pipe structures within an otherwise standard silicon substrate (See Figure 1). This results in a passive fluid cooling system internal to the thin substrate. The resulting substrate is a

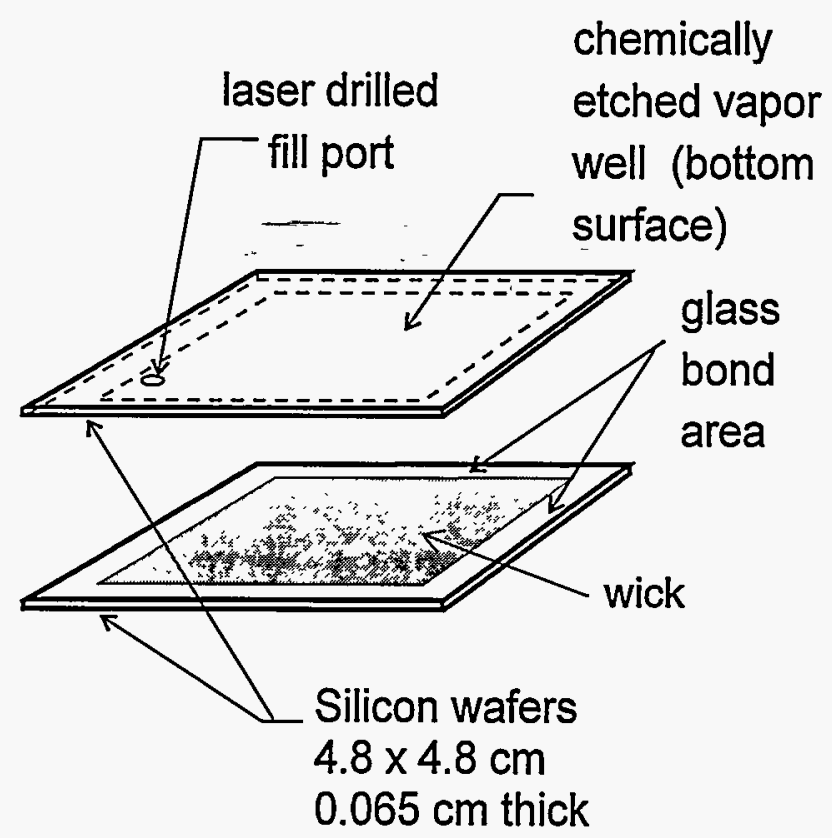

Figure 1. Two silicon wafers are bonded to form a hermetically sealed heat pipe.

large silicon area only twice the standard wafer thickness so that an MCM or power supply module could be built using this silicon (in wafer or rectangular format) as the foundation. With an effective heat conductivity comparable to diamond, the micromachined silicon spreads the heat from a local heat source (eliminates hot spots) and effectively transfers thermal energy to any heat sink in contact. In addition, such a structure still has the coefficient of thermal expansion of silicon; thus, power and temperature cycling pose little threat. Alternatively, the concept could be implemented with a metal sheet chosen with CTE near that of silicon (such as Kovar). The 
microformed embedded heat pipes would enhance the thermal conductivity just as in the silicon development units despite the fact that Kovar's conductivity is only $22 \%$ of silicon conductivity. Since the working fluid conducts most of the thermal energy, the heat pipe efficiency could be high in spite of the lower Kovar conductivity.

Related work performed at Texas A\&M $[2,3]$ uses microchannels etched in the back of the active silicon itself to transfer fluid and enhance heat spreading. Again their system is passive, requiring no active fluid pumping, with the device heat providing the fluid pressure drop. Their micro-channels are v-groves etched within the silicon and sealed with a metal deposition process.

\section{Fabrication History}

Sandia's development started with modeling based on macro-heat pipe formulas and experience. Such modeling suggested that for alcohol working fluid, a wick structure with channels of 50-75 micron dimensions would be optimal. The first units were prepared by sawing 75 micron deep grooves into a wafer's surface using an IC wafer saw. The grooves were center spaced at 150 microns. Some of

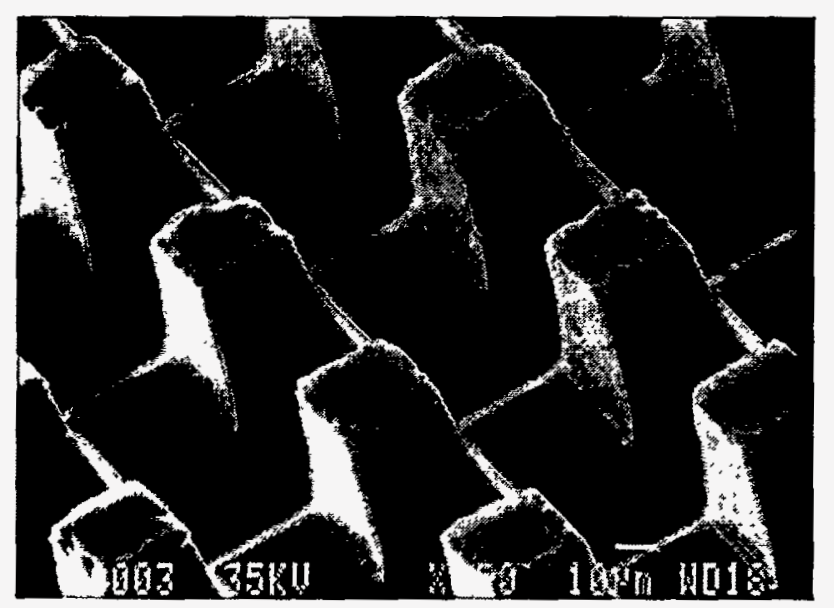

Figure 2. Wick pattern prepared with bidirectional saw cuts on a silicon wafer surface.

the surfaces were grooved in perpendicular directions (See figure 2). Over the last year, as deep etch techniques for silicon micro-machining have become standard, masked etching of more complex wick patterns has become the standard approach (See Figure 3).

Wicking experiments comparing the cooling efficiency of long strips of silicon both with and without wicking patterns were reported in last year's proceedings [4]. These wick evaluation experiments performed with evaporation

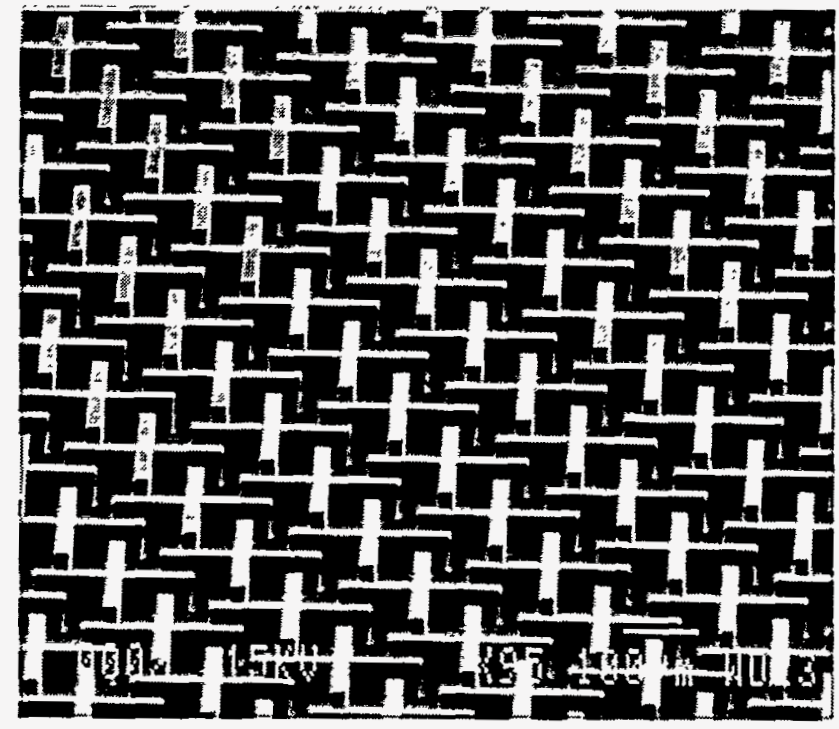

Figure 3. Wick pattern on silicon prepared by a photomask and deep plasma etch technique. The wick features are $25 \mu \mathrm{m}$ wide and $50 \mu \mathrm{m}$ deep.

into air determined the maximum available liquid flow rate that can be driven by surface tension forces. Models of the wick performance and their relation to cooling in heat pipes were developed. Since then the experiments have been repeated in a chamber first evacuated and then equilibrated to the vapor pressure of the working fluid. The results correlated directly to the experiments in air.

To make enclosed micro-heat pipes, the back of an approximately $4.8 \mathrm{~cm}$ square silicon substrate is patterned with a wick structure using a deep anisotropic plasma etching. The boundary of the substrate is left unpatterned to act as the hermetic seal ring for the substrate assembly. The other silicon piece in the assembly is etched using $\mathrm{KOH}$ to produce a shallow well corresponding to the wick area in the substrate. A layer of boro-phosphate silica glass is deposited on this piece. The two pieces can now be wafer bonded together along the seal ring with temperatures of about $800 \mathrm{C}$ for $10 \mathrm{~s}$ of minutes [5]. This seal tests hermetic with standard helium leak checking performed at the most sensitive level.

After the appropriate MCM patterning, or thermal test chip bonding to the substrate, the heat pipe is filled through a small port laser drilled in the substrate. This filling includes evacuation of the embedded heat pipe, filling with a controlled quantity of alcohol or other coolant, and sealing of the assembly.

\section{Testing}

The finished units, one using a working fluid and one running empty as a control, are mounted in the edge- 